\title{
Techn'hom Time Machine : un patrimoine industriel augmenté
}

Marina Gasnier

\section{(2) OpenEdition}

1 Journals

Édition électronique

URL : http://journals.openedition.org/artefact/5967

DOI : 10.4000/artefact.5967

ISSN : 2606-9245

Éditeur :

Association Artefact. Techniques histoire et sciences humaines, Presses universitaires du Midi

\section{Édition imprimée}

Date de publication : 15 juillet 2020

Pagination : 293-299

ISBN : 978-2-8107-0691-4

ISSN : 2273-0753

\section{Référence électronique}

Marina Gasnier, "Techn'hom Time Machine : un patrimoine industriel augmenté », Artefact [En ligne], 12 | 2020, mis en ligne le 21 décembre 2020, consulté le 23 décembre 2020. URL : http:// journals.openedition.org/artefact/5967; DOI : https://doi.org/10.4000/artefact.5967

\section{cc)}

Artefact, Techniques, histoire et sciences humaines est mise à disposition selon les termes de la Licence Creative Commons Attribution - Pas d'Utilisation Commerciale - Pas de Modification 4.0 International. 


\section{Techn'hom Time Machine : un patrimoine industriel augmenté}

\section{Contexte et objectifs}

En accord avec la politique scientifique de l'université de technologie de Belfort-Montbéliard, le projet Techn'hom Time Machine vise à articuler davantage l'enseignement et la recherche, ainsi qu'à développer l'interdisciplinarité entre les sciences humaines et sociales et les sciences de l'ingénierie. À titre expérimental, ce projet envisage le développement de nouvelles formes d'apprentissage. Les enseignants-chercheurs et les étudiants seront co-constructeurs de nouvelles façons de produire la connaissance autour d'un objet historique, en l'occurrence le patrimoine industriel et technique. Idéalement projeté à terme sur l'ensemble de la région nord Franche-Comté afin de restituer le réseau de son maillage industriel de tradition historique, le projet a démarré au semestre de printemps 2019 par une première phase dédiée à la reconstitution numérique spatio-temporelle 3D d'un ancien quartier industriel, aujourd'hui connu sous le nom de Techn'hom, à Belfort ${ }^{1}$. Il s'agit de proposer une histoire totale du quartier en retraçant ses évolutions urbanistique, architecturale, technique, industrielle, démographique et sociale à partir de l'exploitation des archives, d'enquêtes de terrain et de témoignages oraux. L'ensemble sera présenté sur une dalle numérique interactive, démonstrateur placé au sein de l'Open Lab de l'établissement et témoignage du mariage heureux entre

1. Gasnier, 2014.

95 Marina Gasnier, «Techn'hom Time Machine : un patrimoine industriel augmenté », Artefact, 12, 2020, p. 293-299. 
histoire et ingénierie, technologie et patrimoine. En plus de promouvoir les humanités numériques dans un contexte d'analyse et de valorisation de la culture scientifique, technique et industrielle, l'intérêt réside aussi dans son caractère reproductible sur d'autres objets et/ou territoires grâce à une méthodologie qui sera éprouvée tout au long de la phase expérimentale ${ }^{2}$.

\section{Méthodologie et périmètre de l'étude}

Ce projet de restitution multidimensionnelle d'un ancien quartier industriel consiste à investir le champ récent de "l'archéologie industrielle avancée 3 ", c'est-à-dire à partir du présent pour remonter le passé en croisant les sources d'archives et les observations de terrain, ainsi qu'en recourant aux outils numériques. La transformation d'un territoire est le signe même de son évolution, quelle que soit la voie empruntée. Or le développement d'un territoire ne peut se faire dans l'ignorance de son histoire et de ses ressources en place depuis des décennies. Technologiques, scientifiques, humaines, ces ressources sont aussi d'ordre patrimonial dont témoigne Techn'hom comme vecteur de développement économique et scientifique. Ainsi, à partir du croisement des compétences en sciences historiques et en sciences de l'ingénierie, l'idée est de recourir aux humanités numériques pour fabriquer une "machine à remonter le temps » afin de mieux appréhender le tissu industriel et ses artefacts issus de l'histoire des techniques. Les représentations spatiales sont un excellent moyen d'organiser les données et de les visualiser. Elles le sont d'autant plus si on les envisage selon un emboîtement des échelles allant du territoire, au site, à l'entreprise, à l'atelier, au procédé, à la machine, au produit manufacturé, à l'homme. Il s'agit non seulement de capitaliser des informations historiques, non accessibles au plus grand nombre, pour produire une base de données documentaire ; de donner des clés de lecture pour une meilleure compréhension de la construction et de la transformation d'un territoire ; mais aussi d'utiliser ces outils à des fins d'analyse, de valorisation patrimoniale, de vitrine où

2. Afin de profiter de l'expertise extérieure des collectivités, institutions et entreprises partenaires, le projet a fait l'objet d'une convention intitulée : «Patrimoine industriel et numérique. Pour une interdisciplinarité autour des sciences historiques, des sciences de l'ingénierie et des technologies numériques au service de la connaissance, de la recherche et de la valorisation d'objets patrimoniaux dans le nord Franche-Comté ». Elle implique la Région Bourgogne Franche-Comté, le ministère de la Culture, le Territoire de Belfort, Tandem (SEM gestionnaire du site de Techn'hom) et l'UTBM. 3. Laroche, 2007. 
l'histoire et l'innovation sont en regard. Appréhender et promouvoir le patrimoine industriel comme un système sociotechnique à travers les dernières technologies numériques, telle est l'ambition de ce projet.

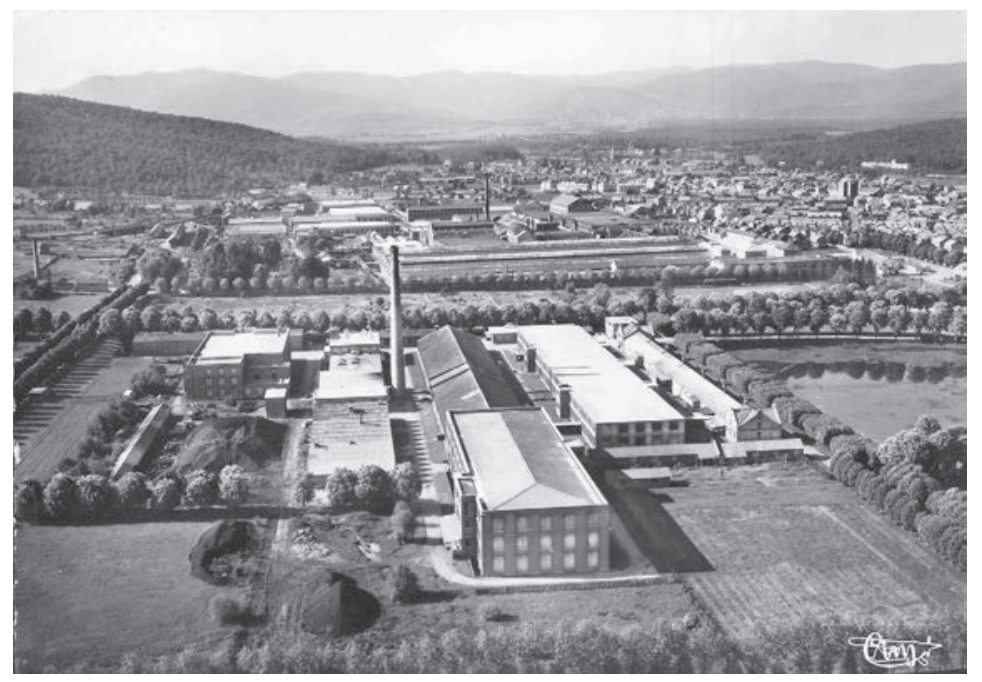

Fig. 1. - Vue aérienne du site

La Filature DMC (premier plan) et l'usine Alsthom (arrière plan), s.d.

\section{Musée de Belfort}

Le premier terrain d'élection du projet se compose, en plus des cités ouvrières et autres infrastructures sociales, de deux anciens sites industriels (la filature Dollfus, Mieg et Cie et la Société alsacienne de constructions mécaniques, devenue Alsthom en 1928) (Fig. 1) ayant fusionné au début des années 2000 pour former Techn'hom : un parc d'activité économique urbain de plus de 100 hectares. En particulier grâce à l'appui des plateformes technologiques (SHERPA, GéoBFC et Nuances ${ }^{4}$ ) de la MSHE Ledoux à Besançon, la création de la base de données destinée à capitaliser

4. GéoBFC est la plate-forme géomatique Bourgogne Franche-Comté commune à la MSHE Ledoux et à la MSH de Dijon. Cette unité met à disposition des chercheurs des équipements et des compétences dont le champ d'action couvre l'ensemble de la chaîne de traitement de l'information spatiale, de l'acquisition de données à la restitution des résultats obtenus par différents traitements et méthodes d'analyse. L'unité NuAnCES (Numérisation et Analyse de Corpus pour la rEcherche Scientifique) permet, quant à elle, la numérisation, la conservation, l'analyse, la diffusion de supports imprimés et d'objets 3D ; elle accompagne les chercheurs en SHS dans leur projet de construction de données partageables et normalisées selon les standards internationaux en mettant à leur disposition différents outils pour la constitution et l'analyse de corpus. 
et à exploiter l'ensemble des ressources, ainsi que les modélisations 3D seront développées par des enseignants-chercheurs et des élèves ingénieurs pour servir au mieux la connaissance d'un territoire historiquement industriel $^{5}$. La démarche sera empirique c'est-à-dire que les outils et la méthodologie devraient évoluer au fil de l'avancée des travaux et des résultats obtenus. Le concept de patrimoine industriel augmenté sera également défini et questionné. Le processus distinguera une phase de collecte des données, de numérisation, de classement, d'analyse et de description des objets, puis de création de métadonnées pour les organiser entre elles. Ce système de gestion des données devra supporter leur hétérogénéité (texte, image, son, vidéo...) et permettre une indexation pour la recherche sémantique. Il convient de préciser que l'objectif vise à dépasser la seule belle animation 3D pour enrichir, voire renouveler la connaissance. La séparation de la base de connaissances de l'interface de consultation est primordiale et présente un double intérêt. D'une part, ce parti pris permet d'assurer l'évolution des contenus du dispositif selon l'apport de nouvelles recherches et/ou préoccupations (et en ce sens ce type de projet est en perpétuelle progression) ; d'autre part, ce type de structuration des données permet d'envisager le développement d'autres interfaces (mobiles, réalité augmentée, etc.) à partir de la même base de connaissances. Un des points complexes réside dans la navigation à l'intérieur de ces données et leurs croisements. Or interroger l'organisation des connaissances s'avère essentiel pour constituer un récit historique. Celui-ci émergera de l'expertise scientifique des chercheurs engagés dans ce projet et du croisement de leurs compétences dans des champs disciplinaires distincts : histoire économique et sociale, cliométrie, histoire des techniques, patrimoine et architecture (CAO sous REVIT - équipe FEMTO-ST/RECITS) ; génie mécanique (numérisation 3D des machines de production des anciens ateliers sous BLENDER - équipe LE2I ; sous CATIA - équipes ICBPMDM-LERMPS et ELLIADD-ERCOS) à partir de sources d'archives et de la mémoire orale ; informatique appliquée : traitement des modèles $3 \mathrm{D}$ et constitution d'une base de données (recherche fondamentale dans le domaine de l'ingénierie des connaissances - équipe LE2I) ; géomatique (géoréférencement des objets architecturaux et techniques - MSHE

5. Daumas, 2006 ; Lamard, 1988 ; Lamard, 1996 ; Lamard, 2001 ; Lamard, Stoskopf, 2009. 
Ledoux) ; ergonomie et graphisme : visualisation d'informations (numérisation 3D et articulation éventuelle avec prototypage rapide - équipe ELLIAD-ERCOS).

\section{Enjeux}

Techn'hom Time Machine recouvre des enjeux pédagogiques, scientifiques et culturels. Il concourt à la promotion de l'interdisciplinarité et de la culture scientifique, technique et industrielle. Au croisement de l'enseignement et de la recherche, il vise d'une part à questionner le processus d'écriture de l'histoire dans une démarche évolutive et collaborative, et d'autre part à développer de nouvelles formes d'apprentissage et d'appréhension de l'histoire. Il conviendra d'analyser le rôle d'un tel traitement dans la construction d'un savoir commun entre le domaine scientifique des ingénieurs et celui des sciences historiques pour une meilleure connaissance et une valorisation des objets patrimoniaux et d'un territoire. Si la réciprocité entre sciences humaines et sociales et sciences pour l'ingénieur est source d'enrichissement, elle l'est aussi en termes de complexité, ne serait-ce que du point de vue sémantique. Toutefois, l'objectif poursuivi est commun : disséquer l'objet, construire le savoir et le valoriser sous une forme renouvelée. Du point de vue de l'histoire des techniques, l'enjeu est également épistémologique. En tentant de formaliser la production technique, l'idée est de s'inscrire dans le sillage de Johann Beckmann ${ }^{6}$ en recourant non pas à la taxinomie comme il l'a fait deux siècles auparavant, mais au maquettage numérique (Fig. 2 et 3). L'ambition est de restituer la génétique des savoir-faire et des pratiques appréhendés sur le temps long de l'histoire. En interrogeant l'interdisciplinarité, comment l'outil informatique peut-il contribuer à analyser l'évolution du geste technique dont le processus relève à la fois de l'intellect, du sensible, de la technique ? Les travaux menés par Nikolai Bernstein au début du Xx ${ }^{e}$ siècle sont autant une source d'inspiration sur le découpage du geste technique?

Même si beaucoup reste à entreprendre dans le domaine des humanités numériques, de l'histoire et du patrimoine ${ }^{8}$, il est désormais convenu que

6. Carnino, Hilaire-Perez, Hoock, 2017.

7. À ce sujet, voir : Bouillon, Guillerme, Mille et al., 2017

8. Ne serait-ce que dans le champ extrêmement vaste et compliqué de l'interopérabilité des données. 
les outils informatiques sont une plus-value en termes de capitalisation de la connaissance, de valorisation et de médiation culturelle. Le recours au produit numérique impacte la perception et l'appréciation du patrimoine. Toutefois, ce projet se veut plus ambitieux. Il vise à amorcer une réflexion épistémologique approfondie pour analyser l'apport de ces outils dans l'étude et la compréhension de l'objet, au-delà de la seule restitution.
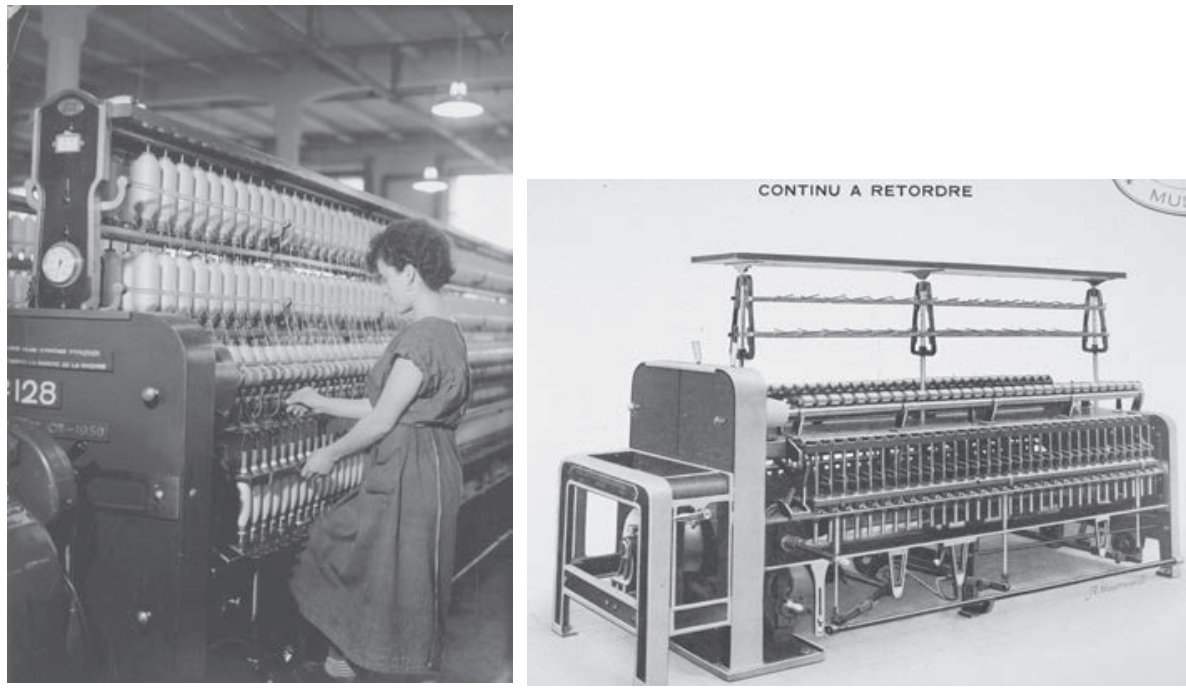

Fig. 2 et 3. - La reconstitution numérique des machines repose sur l'analyse des sources d'archives, tout comme elle recourt à la double mémoire orale et procédurale

À gauche : L'exemple de la Filature DMC, atelier de retordage.

AM Mulhouse, Fonds DMC

À droite : Modèle d'un continu à retordre issu du catalogue de la SACM, Mulhouse.

\section{AM Mulhouse, Fonds SACM}

Enfin en plus de produire un outil de connaissance, l'ambition est de faire valoir l'identité industrielle d'un territoire dont le paysage est encore riche de témoignages. Le site ancestral de Techn'hom en est un bel exemple (Fig. XLVII, cahier couleur). Soumis aux mutations techniques et urbaines, il montre que le patrimoine industriel constitue une véritable ressource territoriale qu'il est nécessaire de promouvoir par-delà nos frontières. Ce projet, tout à fait déclinable sur d'autres territoires, encourage 
ainsi de nouvelles formes de relations avec l'héritage patrimonial tant par l'objet d'étude, que par les technologies déployées.

\section{Marina Gasnier \\ FEMTO-ST/RECITS UMR 6174 CNRS / Université Bourgogne Franche-Comté (UTBM, Belfort)}

\section{Bibliographie}

Bouillon Didier, Guillerme André, Mille Martine et al., Gestes techniques, techniques du geste, Presses universitaires du Septentrion, Lille, 2017.

Carnino Guillaume, Hilaire-Pérez Liliane et Hoock Jochen (dir.), La technologie générale. Johann Beckmann Entwurf der allgemeinen Technologie / Projet de technologie générale (1806), Presses universitaires de Rennes, Rennes, 2017.

Daumas Jean-Claude (dir.), La mémoire de l'industrie. De l'usine au patrimoine, Presses universitaires de Franche-Comté, Besançon, 2006.

GASNIER Marina, "Territorialisation urbaine et processus de patrimonialisation : le cas de Techn'hom à Belfort (Franche-Comté) ", Annales de géographie, n 699, 2014, p. 1168-1192

LAMARD Pierre, Histoire d'un capital familial au XIX' siècle : le capital Japy de 1777 à 1910, Société belfortaine d'émulation, Belfort, 1988.

LAMARd Pierre, De la forge à la société holding Viellard-Migeon et Cie 1796-1996. Polytechnica, Belfort, 1996.

LAMARd Pierre, Sur les traces de l'empire Japy, MTCC, Salins-les-Bains, 2001.

Lamard Pierre et Stoskopf Nicolas (dir.), 1974-1984, une décennie de désindustrialisation?, Picard, Paris, 2009.

Laroche Florent, Contribution à la sauvegarde des objets techniques anciens par l'archéologie industrielle avancée. Proposition d'un modèle d'information de référence muséologique et d'une méthode interdisciplinaire pour la capitalisation des connaissances du patrimoine technique et industriel, thèse de doctorat en génie mécanique, École Centrale de Nantes, vol. I, 2007. 